\title{
Link between periodontal inflammatory disease and Parkinson's disease: Case reports
}

\author{
Ivan Minić $1,{ }^{*}$ Ana Pejčić ${ }^{1}$ and Sanja Popović ${ }^{2}$ \\ ${ }^{1}$ Department of Periodontology and Oral medicine, Medical faculty, University of Nis, Serbia \\ 2 Private praxis "Insta Smile", Niš, Serbia.
}

Publication history: Received on 03 November 2020; revised on 18 November 2020; accepted on 20 November 2020

Article DOI: https://doi.org/10.30574/gscarr.2020.5.2.0096

\begin{abstract}
Parkinson's disease (PD) is caused by a loss of nerve cells in the part of the brain called the substantianigra. Nerve cells in this part of the brain are responsible for producing a chemical called dopamine. The symptoms of Parkinson's disease usually only start to develop when around $80 \%$ of the nerve cells in the substantianigra have been lost. Uncontrolled severe periodontitis caused by periodontal pathogens represents a major infectious threat to the entire body, since released toxins and other inflammatory mediators can reach and affect distant organs. Until now, periodontal disease and Parkinson's disease has been linked only on the basis of poor motor and cognitive control in Parkinson's patient which leads to poor oral health maintenance. Evidence now suggests that chronic neuroinflammation is consistently associated with the pathophysiology of Parkinson's disease. Also, recently, systemic inflammation has been suggested as one of the contributing factors for neurodegeneration. The local inflammatory responses at the site of infection are often linked to CNS disorders such as Parkinson's disease. Infections in the oral cavity, untreated conditions, foci can contribute to the worsening of Parkinson's disease. It is concluded that it is necessary to repair the oral cavity in all patients in order to achieve possible improvement, and on the other hand in these patients it is necessary to fully rehabilitate the oral cavity due to daily functions of the oral cavity which are difficult due to the clinical condition.
\end{abstract}

Keywords: Parkinson's Disease; Periodontal Disease

\section{Introduction}

Parkinson's disease (PD) is caused by a loss of nerve cells in the part of the brain called the substantianigra. Nerve cells in this part of the brain are responsible for producing a chemical called dopamine. Dopamine acts as a messenger between the parts of the brain and nervous system that help control and co-ordinate body movements. If these nerve cells die or become damaged, the amount of dopamine in the brain is reduced. This means the part of the brain controlling movement cannot work as well as normal, causing movements to become slow and abnormal. The loss of nerve cells is a slow process. The symptoms of Parkinson's disease usually only start to develop when around $80 \%$ of the nerve cells in the substantianigra have been lost (1).

It's not known why the loss of nerve cells associated with Parkinson's disease occurs, although research is ongoing to identify potential causes. Currently, it's believed a combination of genetic changes and environmental factors may be responsible for the condition.

PD is one of the most common neurologic disorders, affecting approximately $1 \%$ of individuals older than 60 . The incidence has been estimated to 4.5- 21 cases per 100,000 population per year. Estimates of PD prevalence range from

${ }^{*}$ Corresponding author: Ivan Minić

Department of Periodontology and Oral medicine, Medical faculty, University of Nis, Serbia.

Copyright (C) 2020 Author(s) retain the copyright of this article. This article is published under the terms of the Creative Commons Attribution Liscense 4.0. 
18 to 328 per 100,000 population, with most studies yielding a prevalence of approximately 120 per 100,000 . The incidence and prevalence of PD increase with age. PD is about 1.5 times more common in men than in women. The average age at onset is 60 years, while the onset in persons younger than 40 is uncommon (2).

The most common motor symptoms of PD are tremor (a form of rhythmic shaking), stiffness or rigidity of the muscles, and slowness of movement (called bradykinesia). A person with PD may also have trouble with posture, balance, coordination, and walking. Common non-motor symptoms of PD include sleep problems, constipation, anxiety, depression, and fatigue, among others (3).

Different people experience progression at different speeds, as well. However, physicians have established stages that describe how the disease progresses. These five stages (fig. 1) of Parkinson's are known as the Hoehn and Yahr Scale used by physicians throughout the world to classify patients in research studies $(4,5)$.

\section{Modified Hoehn and Yahr scale}

1.0: Unilateral involvement only
1.5: Unilateral and axial involvement
2.0: Bilateral involvement without impairment of balance
2.5: Mild bilateral disease with recovery on pull test
3.0: Mild to moderate bilateral disease; some postural
instability; physically independent
4.0: Severe disability; still able to walk or stand unassisted
5.0: Wheelchair bound or bedridden unless aided

Figure 1 Stages of Parkinson's disease

The term 'periodontal diseases' encompasses a wide variety of chronic inflammatory conditions of the gingiva (or gums, the soft tissue surrounding the teeth), bone and ligament (the connective tissue collagen fibres that anchor a tooth to alveolar bone) supporting the teeth (6).

Uncontrolled severe periodontitis caused by periodontal pathogens represents a major infectious threat to the entire body, since released toxins and other inflammatory mediators can reach and affect distant organs. During the local inflammatory reaction, inflammatory mediators are produced.The most significant is prostaglandin E2 (PGE2), interleukin-1 $\beta$ (IL-1 $\beta$ ), interleukin 6 (IL-6) and tumour necrosis factor alpha (TNF- $\alpha$ ). Pro-inflammatory cytokines have been distinguished asinflammatory mediators whose production intensity is an indicator of the periodontium tissue destruction activity (7). Patients with periodontal disease have been shown to haveelevated values of these proinflammatory cytokines in the gingival fluid compared to respondents with healthy periodontal tissue (8).

Previously, several studies have emphasized inflammatory responses in the progression of PD and have proposed that chronic conditions and infections, such as diabetes mellitus and periodontal problems, resulting in inflammatory reactions, may be one of the etiological factors in the pathogenesis of $\operatorname{PD}(9,10,11,12)$.

\section{Case reports}

Patient is a man with a 11-year history of PD, who started to show at the age of 54 years Parkinsonian motor symptoms (rigidity, bradykinesia) and later developed "on-off" fluctuations and peak-dose dyskinesias. The patient reported to the clinic due to difficulty swallowing and chewing problems. The first step was to take a detailed medical history from which we came to information about the underlying disease, to be treated for years with Levodopa. He denied other chronic diseases and drug allergies. The patient's neurologist classified his disease into the third degree (mid stage moderate PD) of the disease according to the classificationHoehn and Yahr $(4,5)$. After a detailed medical history, the next step was a clinical examination. Based on the X-ray (Figure 1) and clinical picture, we noticed a large number of residual tooth roots $(11,12,21,22,24,25)$ a total of 6,3 teeth that were indicated for extraction due to large carious destruction $(17,16,45)$ and 9 teeth that required conservative care (Figure 2). 


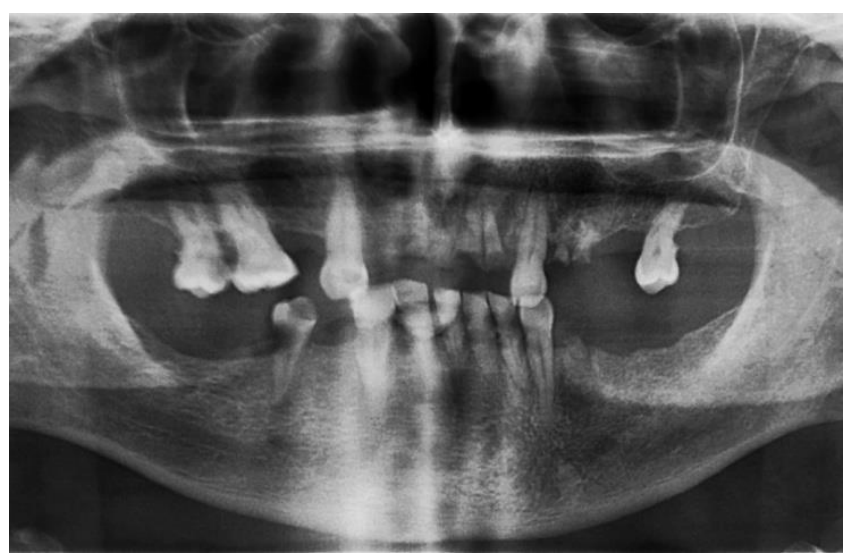

Figure 2 RTG before dental therapy

Periodontal condition was very poor, chronic periodontopathy was diagnosed with pockets over $6 \mathrm{~mm}$ deep on the remaining teeth.

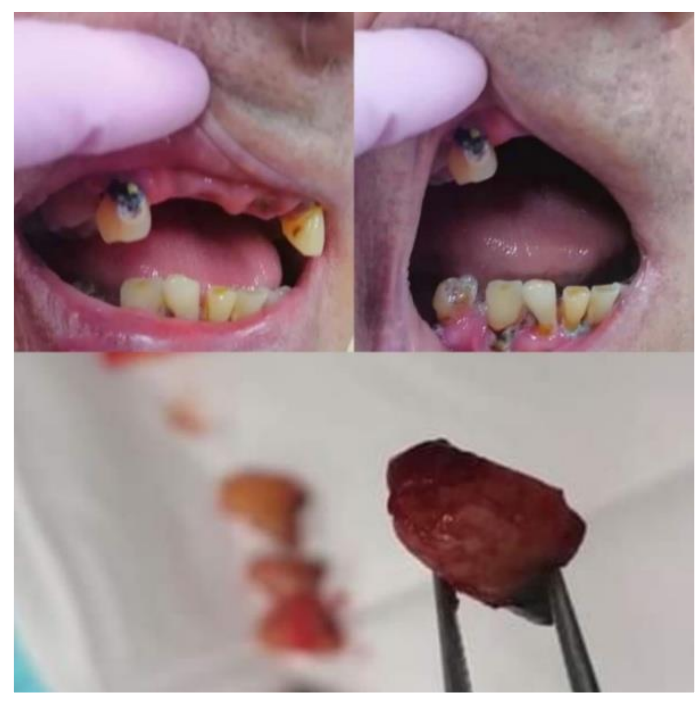

Figure 3 Oral condition before dental therapy

The dental therapy plan was to extract the remaining roots and teeth that were indicated for extraction. This phase of therapy lasted 14 days. After that, conservative treatment of the remaining teeth and periodontal therapy was started, which included removal of soft and hard deposits as well as curettage of periodontal pockets, local rinsing with $3 \%$ hydrogen. Due to severe bleeding and periodontal condition, the patient was prescribed antibiotic therapy with orvagyl antibiotic $400 \mathrm{mg}$ every 8 hours for 14 day. The entire dental treatment lasted 28 days.

At the first control, the patient noticed an improvement in the clinical picture of PD disease, in terms of reduction of subjective symptoms, reduced frequency of tremors and easier mobility. After 2 months from the end of the dental therapy, the control of his neurologist concluded that there was a significant improvement and a new classification from the third to the first degree (mild PD, 1.5 degree) of the disease proves it.For more than six months past, he is still in good condition without any medical treatment.

\section{Discussion}

Parkinson's disease is a chronic progressive neurodegenerative disorder with multifactorial aetiology. Until now, periodontal disease and Parkinson's disease has been linked only on the basis of poor motor and cognitive control in Parkinson's patient which leads to poor oral health maintenance. Evidence now suggests that chronic neuroinflammation is consistently associated with the pathophysiology of Parkinson's disease. Also, recently, systemic inflammation has been suggested as one of the contributing factors for neurodegeneration (13). Several mechanisms 
are known today that may explain the link between periodontitis and Parkinson's disease. During periodontitis, proinflammatory cytokines are produced, including IL-1, IL-6, and TNF- $\alpha$. These cytokines can activate microglial cells, which produce nitric oxide and ROS, leading to death of dopaminergic neurons (14).

From a biological standpoint, the association of periodontitiswith these intricate illnesses may be based on the influence of systemic inflammation and the systemicspreading of periodontal pathogens products with potential affection of brain tissues $(15,16)$. Theseprocesses might support the reported association of PorphyromonasgingivalisIgG levels with compromised delayed memory and calculation (17). Remarkably, a preclinical study in mice showedthat continuous oral application of Porphyromonasgingivalis caused neurodegeneration and formation of extracellular $A \beta 42$ (18).Ebersole and Cappelli (2000) they proved the mediators of inflammation might induce dopaminergic neuronal necrosis or apoptosis, PD initiation and progression, and then cause movement and cognitive disorders (19).

The local inflammatory responses at the site of infection are often linked to CNS disorders such as Parkinson's disease $(20,21)$. The role of the peripheral nervous system is still unclear. Recent studies have shown that the risk of developing PD significantly increases following the occurrence of a peripheral infection, (22) and that the systemic infection could lead to a faster cognitive decline (23). It was even reported that peripheral overexpression of IL-1 $\beta$ accelerated the disease process in an animal model of PD (24).

A study conducted by Cheng-Kai et al., revealed, that patients diagnosed with periodontal disease are 1,43x times more likely to develop Parkinson's disease in the future. Potentially it is caused by inflammatory factors - such as bacterial lipopolysaccharide - synthesis and release of pro-inflammatory mediators IL-1, IL-6, TNF- $\alpha$. Mentioned above factors may activate microglia for the production of reactive oxygen species and nitric oxide, which cause dopaminergic neuron necrosis (25).

Periodontal microorganisms consist of Gram-negative bacteria, which produce the endotoxin lipopolysaccharide. The associated periodontal inflammation could also result in the breakdown of the blood-brain barrier, thereby progressively contributing to the etiology of disabling neurodegenerative disease (26).

\section{Conclusion}

Based on the case report, it can be concluded that the state of oral health is very important for the clinical picture of Parkinson's disease. Infections in the oral cavity, untreated conditions, foci can contribute to the worsening of Parkinson's disease. It is concluded that it is necessary to repair the oral cavity in all patients in order to achieve possible improvement, and on the other hand in these patients it is necessary to fully rehabilitate the oral cavity due to daily functions of the oral cavity which are difficult due to the clinical condition.

\section{Compliance with ethical standards}

\section{Acknowledgments}

Gratitude to all the authors of the text for their great work. Gratitude to the Faculty of Medicine, Department of Dentistry on the occasion provided research.

\section{Disclosure of conflict of interest}

The authors declare no conflicts of interest.

\section{Statement of informed consent}

The patient who participated in the research was informed about the current scientific work and gave a signed consent.

\section{References}

[1] Bernheimer H, Birkmayer W, Hornykiewicz O, Jellinger K, Seitelberger F. Brain dopamine and the syndromes of Parkinson and Huntington. J NeurolSci 1973;20:415-55.

[2] Guttman M, Slaughter PM, Theriault ME, Beboerdap, Naylor CD. Burden of parkinsonism: a population-based study. MovDisord 2003;18:313-9.

[3] Nutt JG, Wooten GF. Diagnosis and initial management of Parkinson's disease. N Engl J Med 2005;353:1021-7. 
[4] National Parkinson Foundation. Accessed online on 12/8/16 at http://www.parkinson.org/understandingparkinsons/what-is-parkinsons/The-Stages-of-Parkinsons-Disease

[5] Parkinson's Resource Foundation. Accessed online on 12/8/16 at http://parkinsonsresource.org/wpcontent/uploads/2012/01/The-FIVE-Stages-of-Parkinsons-Disease.pdf.

[6] Gotsman, I. et al. Periodontal destruction is associated with coronary artery disease and periodontal infection with acute coronary syndrome. J. Periodontol 2007; 78:849-858.

[7] Pradeep AR, Hadge P, Arjun Raju P, Shetty SR, Shareef K, et al. Periodontitis as a risk factor for cerebrovascular accident: a case-control study in the Indian population. J Periodontal Res 2010; 45:223-228.

[8] Deo V, Bhongade ML, Ansari S, Chavan RS. Periodontitis as a potential risk factor for chronic obstructive pulmonary disease: A retrospective study. Indian J Dent Res 2009; 20:466-470.

[9] Ferrari CC, Tarelli R. Parkinson's disease and systemic inflammation. Parkinson's Disease 2011;2011:436813.

[10] Perry VH. Contribution of systemic inflammation to chronic neurodegeneration. ActaNeuropathologica 2010;120:277-286.

[11] Yang YW, Hsieh TF, Li CI, Liu CS, Lin WY, Chiang JH, Li TC, Lin CC. Increased risk of Parkinson disease with diabetes mellitus in a population-based study. Medicine 2017; 96:e5921.

[12] Wu YH, Lee WJ, Chen YH, Chang MH, Lin CH. Premotor symptoms as predictors of outcome in parkinsons disease: a case-control study. PLOS ONE. 2016; 11:e0161271.

[13] Kaur T, Uppoor A, Naik D. Parkinson's disease and periodontitis- the missing link? A review. Gerontology 2016; 33(4):434-438.

[14] Gosney et al. Gosney M, Punekar S, Playfer JR, Bilsborrow PK, Martin MV. The incidence of oral Gram-negative bacteria in patients with Parkinson's disease. European Journal of Internal Medicine. 2003;14:484-487.

[15] Ide M., Harris M, Stevens A, Sussams R, Hopkins V, Culliford D, Fuller J, Ibbett P, Raybould R, Thomas R et al. Periodontitis and cognitive decline in Alzheimer's disease. PLoS One 2016; 11:1-9.

[16] Dominy SS, Lynch C, Ermini F,Benedyk M, Marczyk A, Konradi A, Nguyen M, Haditsch U, Raha D, Griffin C et al. Porphyromonasgingivalis in Alzheimer's disease brains: Evidence for disease causation and treatment with small-molecule inhibitors. Sci. Adv 2019; 5:eaau3333.

[17] Noble JM, Borrell LN, Papapanou PN, Elkind MSV, Scarmeas N, Wright CB. Periodontitis is associated with cognitive impairment among older adults: Analysis of NHANES-III. J. NeurolNeurosurg Psychiatry 2009; 80:1206-1211.

[18] Ilievski V, Zuchowska P K, Green SJ, Toth PT, Ragozzino ME, Le K, Aljewari HW, O’BrienSimpson NM, Reynolds EC, Watanabe K. Chronic oral application of a periodontal pathogen results in brain inflammation, neurodegeneration and amyloid beta production in wild type mice. PLoS One 2018; 13:e0204941.

[19] Ebersole JL, Cappelli D. Acute-phase reactants in infections and inflammatory diseases. Periodontol 2000. 2000;23:19-49.

[20] Wyss-Coray T, Rogers J. Inflammation in Alzheimer disease-a brief review of the basic science and clinical literature. Cold Spring HarbPerspect Med 2012;2:a006346.

[21] Hirsch EC, Vyas S, Hunot S. Neuroinflammation in Parkinson's disease. Parkinsonism RelatDisord 2012;18:S2102.

[22] Dunn N, Mullee M, Perry VH, Holmes C. Association between dementia and infectious disease: Evidence from a case-control study. Alzheimer Dis AssocDisord 2005;19:91-4.

[23] Perry VH, Cunningham C, Holmes C. Systemic infections and inflammation affect chronic neurodegeneration. Nat Rev Immunol 2007;7:161-7.

[24] Godoy MCP, Tarelli R, Ferrari CC, Sarchi MI, Pitossi FJ. Central and systemic IL-1 exacerbates neurodegeneration and motor symptoms in a model of Parkinson's disease. Brain 2008;131:1880-94.

[25] Chen CK, Wu YT, Chang YC. Periodontal inflammatory disease is associated with the risk of Parkinson's disease: a population-based retrospective matched-cohort study. PeerJ. 2017;5:e3647.

[26] Kaur, T.; Uppoor, A.; Naik, D. Parkinson's disease and periodontitis-The missing link? A review. Gerodontology 2016; 33:434-438. 